\title{
Risk of Dementia in First-Degree Relatives of Patients With Alzheimer's Disease and Related Disorders
}

\author{
Richard Mayeux, MD; Mary Sano, PhD; Jenn Chen, PhD; Thomas Tatemichi, MD; Yaakov Stern, PhD
}

\begin{abstract}
- First-degree relatives of patients with Alzheimer's disease (AD) are at greater risk for dementia when compared with the relatives of their healthy peers, but not when compared with the relatives of patients with Parkinson's disease. This may indicate that the risk of dementia in these relatives is not specific to AD or that these studies are biased. We obtained a family history and vital status information on each first-degree relative of patients attending a clinic and in a group of recruited healthy elderly subjects. Patients formed two groups: probable $A D$ and other forms of dementia or cognitive disorders without dementia. The odds of dementia in firstdegree relatives did not differ between patient groups. The odds of dementia in relatives of patients with probable $A D$ or other forms of dementia was six times that in the relatives of the healthy elderly subjects. The cumulative incidence of dementia increased with age in the first-degree relatives of all subjects. Approximately $50 \%$ of the first-degree relatives of patients with $A D$ were demented by age 91 years, but almost the same number of the other patient group's relatives were demented as well. That figure was never reached in the healthy elderly subject's relatives. Because the risk of dementia in first-degree relatives of patients with $A D$ was similar to that for patients with other disorders, we cannot exclude the possibility that this is the result of selection and information biases. Our investigation implies that the increased risk of dementia may not be specific to relatives of patients with $A D$; the risk may also be increased in first-degree relatives of patients with other nourologic disorders.
\end{abstract}

(Arch Neurol. 1991;48:269-273)
Several case-control ${ }^{1-6}$ and cohort studies $^{7.9}$ provide evidence supporting a genetic cause for Alzheimer's disease $(\mathrm{AD})$. The cumulative incidence of an Alzheimer-like dementia in first-degree relatives of patients with $\mathrm{AD}$ may be as high as $50 \%$ by the eighth decade of life..$^{1,2,8-11}$ Although there is a great deal of consistency in those investigations, Farrer et al $^{11}$ did not find an increased risk of dementia in first-degree relatives of patients with $\mathrm{AD}$ compared with the firstdegree relatives of patients with $\mathrm{Par}$ kinson's disease. This indicates that either the increased risk of dementia is not specific to relatives of patients with $\mathrm{AD}$, or that there could be a bias in the way these studies are conducted.

We systematically recorded information about first-degree relatives at the initial visit, before the diagnosis was established, in every patient attending an urban clinic for memory disorders. We estimated the cumulative incidence of dementia in the firstdegree relatives of patients with clinically diagnosed $\mathrm{AD}$ and other disorders, including other types of dementia, and in a group of healthy elderly control subjects. We wanted to determine whether the increased risk of dementia in first-degree relatives of patients with AD is on a genetic basis, and whether this is specific to AD or whether it is due to a selection bias be-

Accepted for publication July 12, 1990

From the Departments of Neurology (Drs Mayeux, Sano, Tatemichi, and Stern) and Psychiatry (Drs Mayeux, Chen, and Stern), Columbia University, College of Physicians and Surgeons, Memory Disorders Clinic, New York State Psychiatric Institute, and the Center for Alzheimer's Disease Research in New York City (NY).

Reprint requests to The Neurological Institute, 710 W 168th St, New York, NY 10032 (Dr Mayeux). cause of heightened awareness in the families of patients with dementia.

\section{PATIENTS, MATERIALS, AND METHODS Subjects}

Patients. -Data from 222 consecutive persons attending a state-supported clinic for memory disorders during a 2-year period in the Washington Heights-Inwood neighborhood of northern Manhattan and the bordering area were used in this study. $\mathrm{Pa}$ tients were referred to the clinic by private physicians in the surrounding area, and about half were self-referred.

Before coming to the clinic, patients were screened in a semi-structured telephone interview with the responsible family member. The purpose of this interview was to exclude people younger than 30 years, or those with a lifetime history of epilepsy or primary psychiatric disorder, such as schizophrenia. Patients with Huntington's disease, stroke, and other major neurologic diseases were also excluded by the telephone interview, where possible, and referred to other clinics.

Healthy Elderly Comparison Group.Elderly adult volunteers were also recruited from the community. A letter was written asking the recipient to participate in a study on aging and memory. We also asked elderly relatives of patients with cerebrovascular disorders in the Presbyterian Hospital, New York, NY, and members of local senior centers to participate. No details about the study hypotheses were provided until after the subjects completed their participation in the study.

\section{Clinical Assessment}

Information regarding medical health, all medications used during the last year, history of their present illness, and both present and past psychiatric records were filed in a standardized recording format. All subjects were rated on their ability to be independent in daily activities.

All patients and healthy elderly control subjects received a physical and neurologic examination. Most patients, and all control 
subjects, received a neuropsychologic test battery. Patients with dementia were hospitalized to undergo a computed tomographic scan of the head, lumbar puncture, electroencephalogram, blood test, and neuropsychologic tests unless these procedures had been performed within the last year and were available for review.

A diagnosis for every patient and recruited subject was made after all clinical studies were completed. Each chart was then independently reviewed by two of us (R.M. and Y.S.) to render a diagnosis. The family history was not used in the diagnostic process at any level. Criteria for primary degenerative dementia were those from the Diagnostic and Statistical Manual of Mental Disorders-III-Revised, ${ }^{12}$ and criteria for Alzheimer's disease were those suggested by the National Institute of Neurological Disorders and Stroke-Alzheimer's Disease and Related Disorders Association Work Group. ${ }^{13}$

\section{Family History}

A semistructured family history questionnaire, similar to published versions ${ }^{14}$ was used in all subjects, patients, and the responsible family members or companions, who acted as informants. The pedigree for all known first-degree relatives was recorded and reviewed at the time of data collection with the responsible informant (attendants or nursing aides were not considered as informants).

The semistructured format for the family history began with standardized questions about each first-degree relative (parents and siblings only; children of probands were excluded) to determine age, sex, position in the family, and the presence of "Alzheimer's disease, dementia, senility, or memory loss." Additional nonstandardized questions were used when needed to clarify the presence and type of dementia. The in. terview was directed to both the patient and the family member (or family members if there was more than one); disagreements between them were resolved before recording the response. The age at death and the cause of death were recorded, as was the current age if still alive when available. When a family reported dementia, followup questions were asked to determine whether the affected relative was still alive or now dead.

Breitner and Margruder-Habib ${ }^{15}$ recommended using a standard time after presumed onset to establish "caseness" and "onset," but we did not believe that the informants or the patients could reliably estimate the date of onset of symptoms in relatives as well as in the proband. Therefore, for all groups we consistently used the current age, if alive, or the age at death reported by the proband's informant to estimate age cumulative incidence in first-degree relatives and avoid an inaccurate estimate of date of "onset." This approach was nondifferential to any group of subjects. The interviewer recording the pedigree was blinded to the final clinical diagnosis of the proband. Records were not used if they were incomplete or if there was some question about the quality of information.

\begin{tabular}{|lc|}
\hline Table 1.-Non-Alzheimer Dementias ${ }^{*}$ \\
\hline \multicolumn{1}{|c|}{ Dementias } & No. \\
\hline Alcohol related & 5 \\
Pick's disease & 3 \\
Major depression with dementia & 3 \\
Head injury & 3 \\
Brain tumor & 2 \\
Focal effects of stroke & 2 \\
Progressive supranuclear palsy & 1 \\
Multiple sclerosis & 1 \\
Creutzfeidt-Jakob disease & 1 \\
Giant cell arteritis & 1 \\
Unknown & 3 \\
\hline N $=25$. &
\end{tabular}

\section{Database Management and Analysis}

All information was entered and stored in a database (CLINFO system). Analysis of variance and statistical methods for rates and proportions were used to compare characteristics of the three groups. ${ }^{16}$ The SAS logistic regression was used to adjust relative-risk estimates, and the SAS product-limit survival estimates were used to estimate the cumulative incidence of dementia in the first-degree relatives. ${ }^{17}$ Power estimates were calculated as suggested by Cohen..$^{18}$

\section{RESULTS Subjects}

Two hundred twenty-two patients were evaluated in the Memory Disorders Clinic (New York, NY) during the 2-year study period. One hundred fourteen patients met the criteria for probable $\mathrm{AD},{ }^{13}$ but only 110 patients had adequate information to provide a pedigree of first-degree relatives. These 110 subjects became the AD patient group.

In the remaining 80 patients, 25 had other forms of dementia as listed in Table 1. In the other 55 patients, other neurologic problems without dementia, based on neuropsychologic testing available, were found in all but $15 \mathrm{pa}$ tients, as noted in Table 2 . Only 68 of these 80 patients had adequate information regarding family pedigree of first-degree relatives. The other 12 were excluded; six were demented and six were nondemented patients. The 68 subjects became the patient comparison group (PCG).

The agreement above chance on the diagnosis of dementia for all subjects and patients by the two clinicians was substantial (for $\mathrm{AD}, \kappa=.73$ and for cognitive disorder and all other patients, $\kappa=.68) .{ }^{36}$ Any disagreement on diagnosis was resolved by two of us (R.M. and Y.S.) and a diagnosis was assigned prior to data analysis. Twenty-eight patients had AD with an associated condition that could have resulted in dementia and were classified with possible AD. ${ }^{13}$ We excluded these patients to be certain about the clinical

\begin{tabular}{|lc|}
\hline \multicolumn{2}{|c|}{ Table 2.-Other Disorders* } \\
\hline \multicolumn{1}{|c|}{ Disorders } & No. \\
\hline $\begin{array}{l}\text { Patients with symptoms but } \\
\text { with no specific disorder } \\
\text { Cognitive impairment due to } \\
\quad \text { stroke }\end{array}$ & 15 \\
$\begin{array}{l}\text { Depression without } \\
\text { dementia }\end{array}$ & 9 \\
$\begin{array}{l}\text { Alcoholism } \\
\text { Amnesia of unknown cause } \\
\text { Cognitive impairment } \\
\quad \text { dementia with head injury } \\
\text { Unclassified psychiatric } \\
\text { disorder } \\
\text { Other disorders with mild } \\
\text { cognitive impairment but } \\
\text { without dementia }\end{array}$ & 7 \\
N $=$ 55. & 3 \\
\hline
\end{tabular}

diagnosis of $A D$ and to avoid $A D$ in the PCG. Since the development of these cohorts the diagnoses in the AD and PCG have not changed over 2 years. Thus, we were confident in our clinical diagnoses of AD and other dementias.

We excluded 68 of the 140 recruited healthy elderly control subjects because the neurologic and neuropsychologic examination indicated some form of intellectual impairment, and 13 others were excluded because of inadequate family history. The remaining 59 healthy elderly subjects formed the healthy elderly group (HEG).

\section{Demographics}

The patients with probable $A D$ and the HEG were similar in age, and both were significantly older than the PCG. The AD and the PCG differed in education, but there was no difference in education between the patients with $\mathrm{AD}$ and the HEG. The probable $\mathrm{AD}$ and HEG cohorts had a similar mean number of first-degree relatives per family, but the PCG had fewer firstdegree relatives than the healthy elderly subjects. Each of these results are presented in Table 3 .

\section{Dementia in First-Degree Relatives}

The relatives of patients with probable AD group were significantly older, which resulted in a greater number of lifetime years at risk than the relatives of either the PCG or the HEG. The number of demented first-degree relatives was significantly higher for the patients with probable $\mathrm{AD}$; there was no difference between the other two groups as is indicated in Table 4.

The estimated odds ratio was compared between groups using logistic regression to adjust for the difference between groups in mean age for firstdegree relatives (Table 5). The adjusted odds associated with dementia in first-degree relatives of the patients with probable $A D$ was six times that of the first-degree relatives of the HEG, 
but only 1.6 times that of the relatives of the PCG. Because the confidence interval included 1 , the odds ratio was not significantly different for the comparison between relatives of patients with probable AD and PCG. When the PCG was divided into those with and without dementia, the adjusted odds ratio associated with dementia in the first-degree relatives of the patients with probable AD was still no different than that calculated for the relatives of either portion of the PCG (demented and nondemented).

Because there was no difference between these two groups of first-degree relatives ( $A D$ and $P C G$ ), we evaluated the possibility of a type II error. Assuming the log odds ratio for this comparison was the same as that seen in $A D$ vs $H E G$ analysis (log odds ratio $=1.8, \mathrm{SE}=0.3$ ) and an $\alpha$ level of .05 , we calculated the power of the test to be $99 \%$. Thus, the likelihood of a type II error in accepting no difference in odds ratios between first-degree relatives of patients with $A D$ and those of the PCG was less than $1 \% .{ }^{17}$

The adjusted odds ratios were also calculated for the demented and nondemented subjects in the PCG and compared with the HEG as noted in Table 5. The odds of dementia in the relatives of the demented PCG was six times that of the HEG. The odds of dementia in the relatives of the nondemented PCG was four times that of the HEG.

Product-limit survival analysis was used to estimate the cumulative incidence of dementia in first-degree relatives of the three groups. Cumulative incidence of dementia in the three groups increased with age as illustrated in the Figure. By age 91 years, $50 \%$ of the first-degree relatives of the probable $A D$ group were considered demented. However, by the same age only $20 \%$ of the first-degree relatives of the HEG had developed dementia. Remarkably, by age 91 years, nearly $40 \%$ of the first-degree relatives of the PCG were considered demented. The cumulative incidence of dementia in the first-degree relatives of patients with $A D$ and the PCG was significantly higher than that in the HEG (log rank test $\left.\chi^{2}=27.48, P<.001\right)$. The cumulative incidence in these two groups (AD and PCG) did not differ statistically.

\section{COMMENT}

Our data confirm earlier studies ${ }^{1-10}$ indicating increased risk of dementia in the first-degree relatives of patients with $\mathrm{AD}$ when compared with the first-degree relatives of their healthy

Table 3.-Age, Years of Education, and Number of First-Degree Relatives in all Subjects *

\begin{tabular}{|cccc|}
\hline Subject Group & Age, $y$ & Education & No. of First-Degree Relatives \\
\hline Probable AD $(n=110)$ & $\begin{array}{c}71.1(10.3) \\
\text { (range, } 48-89)\end{array}$ & $12.2(4.3)$ & $5.2(2.3)$ \\
\hline PCG $(n=68)$ & $\begin{array}{c}65.7(11.6) \dagger \\
\text { (range, 34-89) }\end{array}$ & $13.9(4.6) \neq$ & $4.6(2.1) \S$ \\
\hline HEG $(n=59)$ & $\begin{array}{c}70.4(6.9) \\
\text { (range, } 60-90)\end{array}$ & $11.7(3.9)$ & $5.7(2.9)$ \\
\hline
\end{tabular}

"Probable Alzheimer's disease (AD) represents the patients with clinically diagnosed AD; patient comparison group (PCG), all of the other patients listed in Tables 1 and 2; healthy elderly group (HEG), recruited healthy elderly cohort; and numbers within parentheses, mean values with SDs.

tPatient comparison group was younger, $F=6.4, P<.01$.

¥Patient comparison group had more education, $F=4.4, P<.05$

$\S$ Patient comparison group had fewer first-degree relatives, $F=3.6, P<.05$

\begin{tabular}{|cccc|}
\hline \multicolumn{4}{|c|}{ Table 4.-Mean Age of First-Degree Relatives of All Subjects and Percent } \\
Considered Demented*
\end{tabular}

"Probable Alzheimer's disease (AD) represents the patients with clinically diagnosed AD; patient comparison group (PCG), all of the other patients listed in Tables 1 and 2; healthy elderly group (HEG), recruited healthy cohort; $\mathrm{N}$, total number of first-degree relatives; and numbers within parentheses are mean values with SDs. tRelatives of probable $A D$ group were older, $F=4.36, P<.05$.

¥Percent of first-degree relatives demented was greater in probable AD group, $\chi^{2}=14.9, P<.001$.

\begin{tabular}{|c|c|c|}
\hline \multicolumn{3}{|c|}{$\begin{array}{l}\text { Table 5.-Estimated Odds Ratios (Adjusted for Age of the First-Degree Relatives) for } \\
\text { Dementia in First-Degree Relatives of Patients With AD, the Patient Comparison Group, } \\
\text { and the Healthy Elderly Group* }\end{array}$} \\
\hline Subjects & Odds Ratios & $95 \% \mathrm{Cl}$ \\
\hline Probable AD vs combined patient comparison group & 1.58 & $0.88,2.86$ \\
\hline Probable AD vs patient comparison group with dementia & 0.95 & $0.42,2.17$ \\
\hline Probable AD vs patient comparison group without dementia & 1.96 & $0.95,4.85$ \\
\hline Probable $A D$ vs healthy elderly group & 6.19 & $2.804,13.69$ \\
\hline $\begin{array}{l}\text { Patient comparison group with dementia vs healthy elderly } \\
\text { group }\end{array}$ & 6.12 & $2.073,18.10$ \\
\hline Without dementia vs healthy elderly group & 3.95 & $1.38,11.35$ \\
\hline
\end{tabular}

"AD indicates Alzheimer's disease; $\mathrm{Cl}$, confidence interval.

peers. However, we have also found that other neurologic disorders, even those without dementia, may be associated with almost the same degree of risk to their first-degree relatives. The odds for dementia in the first-degree relatives of patients with $A D$ and the demented patients in the PCG were both six times that for relatives for the HEG after adjusting for age differences. Even the relatives of the nondemented members of the PCG had four times the risk of the relatives of the HEG.

There are two major issues to consider in the interpretation of our results: validity, which includes selection and information bias, and specificity.

\section{Selection Bias}

Most case-control studies, ${ }^{1 \cdot 7}$ except for the population-based study of
Hofman et al,' have identified patients with AD who voluntarily attend a hospital or clinic and have then recruited a healthy individual to represent a comparison cohort. Patients, or their family members in the case of $\mathrm{AD}$ or another dementia, may remember their family history better than the recruited healthy elderly persons resulting in what Rocca and Amaducci ${ }^{19}$ termed an awareness bias. A family history of dementia would be a strong motive for seeking medical advice, and would increase the possibility of identifying heredity as a risk factor. Thus, the increased risk in many of the casecontrol studies of AD could be due to the selection of families with an enhanced knowledge of their family history. The relatives of our PCG may have been influenced by this same bias. Selection bias may also help to explain 


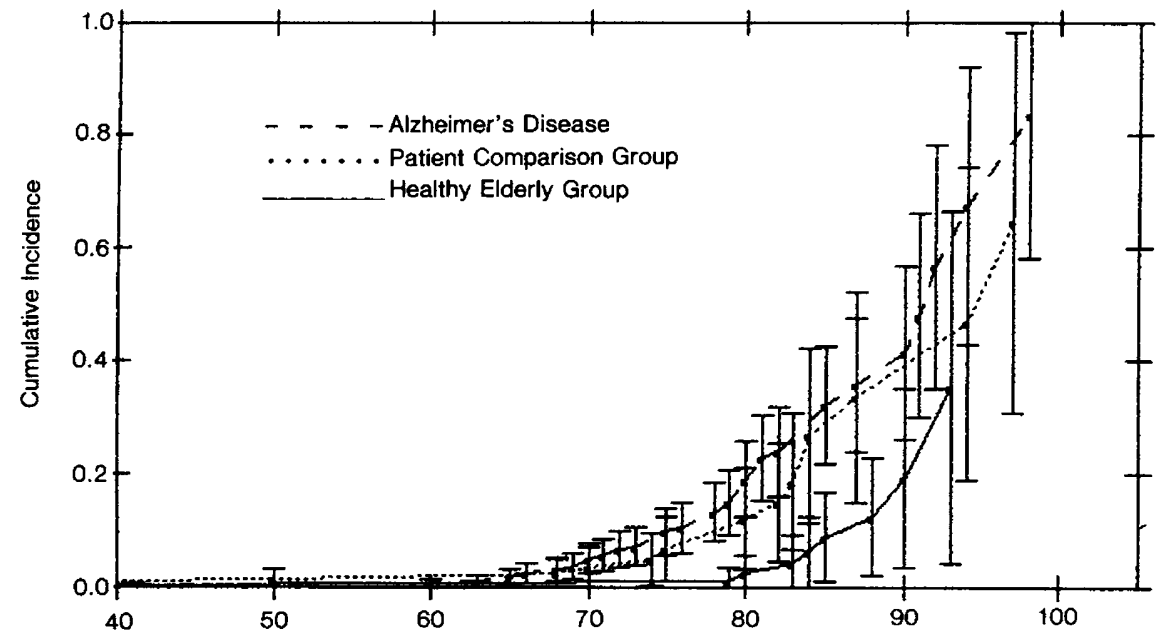

Reported Age or Age-at-Death of First-Degree Relatives

Analysis showing the cumulative incidence of dementia among first-degree relatives of either patients with Alzheimer's disease, subjects from the patient comparison group, or members of the healthy elderly group. The hatched line indicates relatives of patients with Alzheimer's disease; dotted line, relatives of the patient control group; straight line, healthy elderly subjects' relatives. By the age of 91 years, the cumulative incidence of dementia was $48.7 \%$ in the relatives of pa. tients with Alzheimer's disease, but about $38.9 \%$ in the patient comparison groups and less than $23 \%$ in the relatives of the healthy control subjects.

why the risk exceeded $50 \%$ in some $\mathrm{AD}$ families in another study by Farrer et al, ${ }^{10}$ although the risk in excess of $50 \%$ was interpreted as an indication of an associated environmental factor.

We were unable to obtain family history data from $15 \%$ of the PCG and $18 \%$ of the HEG compared with $3.5 \%$ of the AD group, which could lead to an awareness bias. The inability to obtain information about some of the firstdegree relatives in two of the groups could result in a differential misclassification of cases in these families and might lead us to reject or favor the "null hypothesis" of no difference in risk to first-degree relatives of patients with AD. ${ }^{20,21}$ However, this would not explain the difference in risks for relatives between these two comparison groups. Although differential bias may have been present in the comparison of $\mathrm{AD}$ with the $\mathrm{HEG}$, we believe that our use of a cohort of patients attending the same clinic lessened the impact of awareness bias among control subjects.

\section{Information Bias}

A second, separate family history interview was not performed by us, but we consider the data quality to be as valid as that collected by others because our interview format was similar..$^{1-8}$ As in all previous studies, and ours, information was obtained in interviews with other family members. Few "affected" family members have actually been examined in these and Marder et $\mathrm{al}^{23}$ have found dementia and Parkinson's disease to aggregate in first-degree relatives of patients with AD or Parkinson's disease, this lack of difference in risk might have been anticipated. Similarly, we may have introduced this type of problem by using patients with other neurologic disorders as a control group. The lack of difference in risk of dementia to first-degree relatives in the study by Farrer et al ${ }^{11}$ and our own, could also indicate that the increased risk of dementia in first-degree relatives is not specific to $A D$ and may be present in a number of neurologic diseases.

We did not attempt to estimate age at onset of dementia in the relatives because so few informants could provide that information. Farrer et $a^{10}$ "censored" this type of age estimate, noting that such estimates increase the age at onset. Although our approach was nondifferential to group, estimating the correct age at onset would result in a shift of the cumulative risk curve to the left by as much as 8 years. A review of the Figure, based on this left shift, would not alter our observations regarding the similarity in risk between the $A D$ and $P C G$ relatives, but would have the effect of lowering the age at which risk approaches $50 \%$.

We were cautious in our interpretation of the maximum estimates of cumulative risk from our data. Maximum estimates from cumulative incidence curves produce large standard errors as is indicated in our figure, particularly for the very old ages where the number of survivors diminish. Thus, beyond the age of 90 years, maximum estimates may not be as accurate.

We have found that issues of validity and specificity have considerable impact on the interpretation of cumulative risk and the calculation of odds ratios in the first-degree relatives of these patients. While our data do not lessen the likelihood that heredity plays a role in the cause of $\mathrm{AD}$, they do imply that other forms of dementia and other cognitive disorders can produce similar risks in first-degree family members. Therefore, other risk factors must be investigated in the cause of $\mathrm{AD}$.

This work was supported by federal grants, AG07232, NS26179, RR00645, the Charles S. Robertson Memorial Gift for Alzheimer's disease, and federal grant PO-50-AG-08702 to the Center for Alzheimer's Disease Research in New York City (NY).

We thank Allan Hauser, MD, Karen Marder, $\mathrm{MD}$, and Ruth Ottman, PhD, for their help with the interpretation of our data and assistance with the manuscript. 


\section{References}

1. Breitner JCS, Silverman JM, Mohs RC, Davis KL. Familial aggregation in Alzheimer's disease: comparison of risk among relatives of early- and late-onset cases, and among male and female relatives in successive generations. Neurology. 1988:38:207-212.

2. Huff FJ, Auerbach BA, Charkravarti A, Boller $F$. Risk of dementia in relative of patients with Alzheimer's disease. Neurology. 1988;38:786-790.

3. Shalat SL, Seltzer B, Pidcock C, et al. Risk factors for Alzheimer's disease: a case-contro study. Neurology. 1987;37:1630-1633.

4. Chandra V, Philipose V, Bell PA, Lazaroff A Schoenberg BS. Case-control study of late-onset "probable Alzheimer's disease." Neurology. 1987; 37:1295-1300.

5. Amaducci LA, Fratiglioni L, Rocca WA, et al Risk factors for clinically diagnosed Alzheimer' disease: a case-control study of Italian population. Neurology. 1986;36:922-931.

6. Graves AB, White D, Koepsell T, Reifler B. A case-control study of Alzheimer's disease. $A m$ Epidemiol. 1987;126:754.

7. Heyman A, Wilkinson WE, Hurwitz BJ, et al Alzheimer's disease: genetic aspects and associated clinical disorders. Ann Neurol. 1983;14:507515.

8. Breitner JCS, Murphy EA, Silverman JM,
Mohs R, Davis KL. Age-dependent expression of familial risk in Alzheimer's disease. Am J Epidemiol. 1988;128:536-548.

9. Hofman A, Schulte W, Tanja TA, et al. History of dementia and Parkinson's disease in 1stdegree relative of patients with Alzheimer's disease. Neurology. 1989;39:1589-1592.

10. Farrer LA, Meyers RH, Cupples LA, et al. Transmission and age-at-onset patterns in familial Alzheimer's disease: evidence for heterogeneity. Neurology. 1990;40:395-403.

11. Farrer LA, 0'Sullivan DM, Cupples LA, Growdon JH, Myers RH. Assessment of genetic risk for Alzheimer's disease among first-degree relatives. Ann Neurol. 1989;25:485-493.

12. American Psychiatric Association. The Diagnostic and Statistical Manual of Mental Disorders-Revised. 3rd ed. Washington, DC: American Psychiatric Association; 1986.

13. McKhann G, Drachman D, Folstein M, et al. Clinical diagnosis of Alzheimer's disease: report of the NINCDS-ADRDA Work Group under the auspices of the Department of Health and Human Services Task Force on Alzheimer's disease. Neurology. 1984;34:939-944.

14. Breitner JCS, Folstein MF. Familial Alzheimer dementia: a prevalent disorder with specific clinical features. Psychol Med. 1984;14:63-80.
15. Breitner JCS, Margruder-Habib KM. Criteria for onset critically influence the estimation of familial risk in Alzheimer's disease. Genet Epidemiol. In press.

16. Fleiss JL. Statistical Methods for Rates and Proportions. New York, NY: John Wiley \& Sons Inc; 1981

17. SAS. User's Guide: Statistics. 5th ed. Cary NC: SAS Institute; 198

18. Cohen J. Statistical Power Analysis for the Behavioral Sciences. Philadelphia, Pa: Lea \& Febiger; 1988:215-252.

19. Roeca WA, Amaducci LA, Schoenberg BS Epidemiology of clinically diagnosed Alzheimer's disease. Ann Neurol. 1986;19:415-424.

20. Copeland KT, Checkoway H, McMichael AJ, Holbrook RH. Bias due to misclassification in the estimation of relative risk. Am $J$ Epidemiol. 1977;105:488-495.

21. Rothman KJ. Modern Epidemiology. Boston, Mass: Little Brown \& Co Inc; 1986:80-86.

22. Kupper LL. Effects of the use of unreliable surrogate variables on the validity of epidemiologic research studies. Am J Epidemiol. 1984; 120:634-638.

23. Marder K, Flood P, Cote L, Mayeux R. A pilot study of risk factors for dementia in Parkinson's disease. Mov Disord. 1990;5:156-161. 